INPLASY

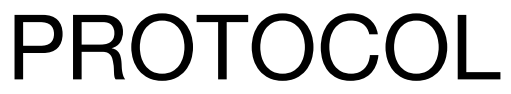

To cite: Irwin et al. Treatmentrelated changes of molecular biomarkers in major depressive disorder: a systematic review and metaanalysis. Inplasy protocol 2021100105. doi: 10.37766/inplasy2021.10.0105

Received: 27 October 2021

Published: 27 October 2021

Corresponding author:

Sandra Carvalho

sandrarc@ua.pt

Author Affiliation:

University of Aveiro

Support: FCT - PTDC/PSI-

ESP/29701/2017.

Review Stage at time of this submission: Data analysis Completed but not published.

Conflicts of interest:

None declared.

\section{Treatment-related changes of molecular biomarkers in major depressive disorder: a systematic review and meta-analysis}

Irwin, CL1; Coelho, PS², Kluwe-Schiavon, B33 Silva-Fernandes, A4; Gonçalves, ÓF5; Leite, J6; Carvalho, S7.

Review question / Objective: The aim of this review is twofold: first, we sought to identify candidate biomarkers that could provide information on whether an individual with MDD would respond positively to common non-pharmacological treatments, and secondly, to conduct a meta-analysis to determine whether one form of common nonpharmacological treatment (namely CBT, tDCS and TMS) would produce better results over another in regards to its influence on biomarker levels.

Information sources: The information sources used were: three online databases (PubMed, Scopus, and PsycINFO) to identify English-language human randomised controlled trials unrestricted by year of publication.

INPLASY registration number: This protocol was registered with the International Platform of Registered Systematic Review and Meta-Analysis Protocols (INPLASY) on 27 October 2021 and was last updated on 27 October 2021 (registration number INPLASY2021100105).

\section{INTRODUCTION}

Review question / Objective: The aim of this review is two-fold: first, we sought to identify candidate biomarkers that could provide information on whether an individual with MDD would respond positively to common non-pharmacological treatments, and secondly, to conduct a meta-analysis to determine whether one form of common non-pharmacological treatment (namely CBT, tDCS and TMS) would produce better results over another 
in regards to its influence on biomarker levels.

Rationale: Understanding the effect of biomarkers on MDD could be advantageous in several ways as: a) adjunctive biomarkers for improving diagnosis, b) a measure of disease progress, c) a prognostic tool, or d) a means of predicting or following response to a certain treatment. In the context of therapeutic intervention trials, biomarkers are beneficial to clinicians as they can theoretically provide objective and externally measured information about the outcome of a therapeutic intervention on a participant (e.g., benefit/harm or lack of benefit/harm) preceding the intervention itself. However, currently there are no validated biomarkers for MDD, which hinders clinicians' ability to offer precise and personalised medicine to affected individuals that could optimize symptom remission and increase response rate while simultaneously minimising the likelihood of harmful side effects. While recent studies and systematic reviews have investigated biomarkers in predicting MDD treatment response, to our knowledge no study has directly compared these biomarkers exclusively across non-pharmacological treatment classes. Chemical biomarker predictors of differential treatment response would be especially useful in promoting quick, long-lasting remission in affected individuals, and novel findings could be used to support different hypotheses of MDD pathogenesis and/or shed light on the mechanisms of action across treatment classes.

Condition being studied: The inclusion criteria for this systematic review were the following: (1) Population: Studies conducted in adult participants (18 years of age or older) with at least one group of participants having MDD as a main diagnosis (as defined by recognized diagnostic criteria); Studies in which participants were taking none or were on stable medication preceding the start of the treatment; (2) Intervention(s): CBT, tDCS, or TMS treatments conducted repetitively (more than one session); CBT therapy could be offered in any format (e.g., in-person, online, phone, in groups, etc.), so long as a certified psychologist or psychiatrist was leading the treatments); (3) Assessment(s): Studies that reported chemical biomarker data both pre- and post-treatment; (4) Study design: Parallel, randomized controlled trials (RCTs) published in a peer reviewed journal.

\section{METHODS}

Search strategy: This systematic review was conducted according to the guidelines outlined by the Preferred Reporting Items for Systematic Reviews and Meta-analyses (PRISMA) statement. An initial article search was conducted in October 2020 exclusively within three online databases (PubMed, Scopus, and PsycINFO) to identify English-language human randomized controlled trials unrestricted by year of publication. Each search query consisted of keywords and subject headings for the following terms: "Major depressive disorder", "MDD", "major depression", “depressed", "depressive", "biomarkers", "blood", "fecal", "microbio*”, "MRS", "magnetic resonance spectroscopy", "immun*", "hormon*", "metabolic*", "neuroendocrin*", "neurotransmit"”, "protein*”, "neurotroph", "gastrointestin*", "proteom*", "plasma", "biomarker*", "marker*", "surrogate*", "serum", "saliva", "urine", "cerebrospinal", "PET", "ELISA", "positron emission", "cognitive behavioral therapy", "CBT", "cognitive behav*", "transcranial direct current stimulation*", "transcranial magnetic stimulation*", "tDCS", and "TMS". Slight adjustments were made to the search query according to the syntax specifications of each database. In July 2021, a top-up search was conducted to determine whether any new studies had been published since the initial search.

Participant or population: People with Major Depression Disorder.

Intervention: Non Pharmacological Treatments: Cognitive Behavior Therapy, Trasncranial Direct Current Stimulation and Transcranial Magnetic Stimulation. 
Comparator: Baseline versus pós treatment; active versus sham intervention; non pharmacological treatment (CBT, tDCS and TMS) versus other treatment options.

Study designs to be included: Parallel, randomized controlled trials (RCTs).

Eligibility criteria: The inclusion criteria for this systematic review were the following: (1) Population: Studies conducted in adult participants (18 years of age or older) with at least one group of participants having MDD as a main diagnosis (as defined by recognized diagnostic criteria); Studies in which participants were taking none or were on stable medication preceding the start of the treatment; (2) Intervention(s): CBT, tDCS, or TMS treatments conducted repetitively (more than one session); CBT therapy could be offered in any format (e.g., in-person, online, phone, in groups, etc.), so long as a certified psychologist or psychiatrist was leading the treatments); (3) Assessment(s): Studies that reported chemical biomarker data both pre- and post-treatment; (4) Study design: Parallel, randomized controlled trials (RCTs) published in a peer reviewed journal. The following exclusion criteria were applied: (1) Population: Studies conducted in children or adolescents (under 18 years of age), animals, exclusively in vitro experiments, or post-mortem samples; Studies with individuals whose main diagnosis was not MDD, who were disclosed to have a past or present systemic disease/disorder (e.g., diabetes, cardiovascular disease) that was the focus of the study, who were pregnant, who had suffered a brain injury, who were a member of a risk group that was the focus of the study, whose depressive symptoms were said to have originated from another physical condition or mental disorder (e.g., postpartum depression), or individuals diagnosed with a personality disorder; Studies in which participants were reported to be taking unstable medication doses or reported to be dependent on or abusing alcohol and/or illicit drugs; (2) Intervention(s): Studies conducting treatment combinations on the same participant(s) (e.g., TMS and then CBT) if the data from a control group (i.e., group that only received one treatment) was not reported; (3) Assessment(s): Studies that assessed biomarker data using other methodologies that do not directly measure the levels of a specific chemical biomarker marker (e.g., fMRI, EEG) were excluded, unless complementary chemical biomarker data were reported; Studies that exclusively reported genetic markers (e.g., polymorphism profile); (4) Study design: All other non-compliant study designs (e.g., pilot studies, preliminary studies, cohort studies, case-control studies, reviews, qualitative analyses, meta-analyses, or grey literature, duplicated datasets, etc.).

Information sources: The information sources used were: three online databases (PubMed, Scopus, and PsycINFO) to identify English-language human randomised controlled trials unrestricted by year of publication.

Main outcome(s): The main outcome of the review was the magnitude of the effect of NIBS on the change of biomarker levels when compared to sham/placebo conditions and other treatments. Hence, we considered the standardized mean difference of the magnitude of biomarker change (i.e., endpoint level minus baseline level) as a dependent variable using the maximum likelihood estimator method. Due to a small number of studies that measured the same biomarker, we performed four independent meta-analyses for each of the following biomarker's classes: all interleukins, pro-inflammatory interleukins, anti-inflammatory interleukins, and neurotrophic. Additionally, three independent meta-analyses were performed: one for BDNF, one for IL-6, and one for TNF-a, since these biomarkers were investigated in at least three studies each. As clustering biomarkers by biological class may have some methodological implications, a random effect for biomarker type was included in the models.

Data management: The mean and the standard deviation were recorded for the baseline and endpoint biomarker levels 
from both intervention and control conditions. If instead of mean and standard deviation, other values were reported (i.e., median, standard error, or interquartile range), the mean and standard deviation were estimated as follows: (a) median as mean; (2) standard error multiplied by the square root of sample size as standard deviation; and (3) interquartile range divided by 1.35 as standard deviation. All analyses were performed using the package "metafor" (version 2.0-0). from the open-source statistical software R (version 3.4.3).

Quality assessment / Risk of bias analysis: Two authors screened the titles and abstracts of articles from the primary search independently against inclusion and exclusion criteria. The full text of qualifying articles was then assessed against the same standard. Any discrepancies were resolved first through discussion amongst themselves, and if a consensus could still not be reached, by conferring with other group members. Following PRISMA guidelines, the quality assessment of selected studies and the data extraction were conducted independently by two authors, first separately and then together. The Jadad scale was used to assess the methodological quality of selected clinical trials. All the inter-rater agreements between authors were verified prior to resolving disagreements. The inter-rater reliability between authors, measured using Cohen's Kappa coefficient, was 0.957 $(K=95.7 \%)$ and $0.824(K=82.4 \%)$ for the fulltext classification and Jadad methodological assessment, respectively.

Strategy of data synthesis: The mean and the standard deviation were recorded for the baseline and endpoint biomarker levels from both intervention and control conditions. If instead of mean and standard deviation, other values were reported (i.e., median, standard error, or interquartile range), the mean and standard deviation were estimated as follows: (a) median as mean; (2) standard error multiplied by the square root of sample size as standard deviation; and (3) interquartile range divided by 1.35 as standard deviation.
Moreover, the following data was extracted from each selected study: study groups (i.e., type(s) of intervention and control), percentage of female participants, mean age, description of MDD diagnosis, drug status to treat MDD diagnosis (i.e., drugnaive, stable medication, tapered medication), treatment duration, treatment frequency, total number of sessions, session duration, time points (i.e., symptomatology assessment and biomarker collection), main depressive symptomatology assessment used over trial (e.g., MADRS), description of clinical change over trial (i.e., no change, increase, or decrease in symptom severity) and all reported biomarker level data compared within and between the treatment groups covered under the scope of this review (i.e., no change, pre-to-post change within/ between treatment arms, and directionality of change).

Subgroup analysis: Since we defined several categories of control conditions (i.e., sham/placebo, active interventions such as cognitive behaviour therapy and psychopharmacological treatments), our meta-analytical models were performed as follows: a comparison of the NIBS with sham/placebo conditions, followed by a comparison of the NIBS with active interventions as well as with psychopharmacological treatments. In this way, we first compared the NIBS against possible placebo effects and, afterwards, against other treatments.

Sensitivity analysis: Heterogeneity was measured by both Cochran's $Q$ and Isquared indices when calculated for all models. Study bias and influential cases were investigated by examining the standardized residual for each study and checking for outliers. The influence of studies that had a z-scores of greater than \pm 1.96 was examined using the "leave one out" method. Thus, the results of our metaanalysis were recalculated $n-1$ times, each time leaving out one possible influential study. If in this sensitivity analysis, the studies did not substantially change the overall effect size, we opted to retain them in the overall analyses. Publication bias 
was assessed by visually inspecting funnel plots and by calculating Rosenthal's failsafe $N$, which calculates the number of studies averaging null results that would have to be added to the given set of observed outcomes in order to reduce the combined significance level ( $p$-value) to a target alpha level (e.g., 0.05).

Language: English.

Country(ies) involved: Portugal.

Keywords: Major depressive disorder, Biomarkers, Non-pharmacological treatments, Cognitive behavioral therapy, Non-invasive brain stimulation interventions, Neurotrophic growth factors.

Dissemination plans: Results of the study will be presented in scientific meetings, as well as in sessions to the general public. Data will be stored in public data repositories and available to the broader scientific community.

Contributions of each author:

Author 1 - Courtney L. Irwin - Main contributing author for the first draft of the the manuscript, data extraction, and data analysis.

Email: cirwi095@uottawa.ca

Author 2 - Patrícia S. Coelho - Main contributing author for the first draft of the manuscript, data extraction, and data analysis.

Email: patricia.a.soares.coelho@gmail.com Author 3 - Bruno Kluwe-Schiavon Contributed to the first draft of the manuscript, data extraction, and data analysis.

Email: bschiavon@psi.uminho.pt

Author 4 - Anabela Silva-Fernandes Contributed to the first draft of the the manuscript, data extraction, and data analysis.

Email: asfernandes@psi.uminho.pt

Author 5 - Óscar F. Gonçalves - Contributed to the design of the study protocol, contributions to the final draft of the manuscript.

Email: oscar@fpce.uc.pt

Author 6 - Jorge Leite - Design of the study, data selection and statistical analysis, contributions to the final draft of the manuscript.

Email: jorgel@mail.upt.pt

Author 7 - Sandra Carvalho - Principal investigator of the funded study, design of the protocol, data selection, contributions to the final draft of the manuscript.

Email: sandrarc@ua.pt 